\title{
Comparative study of Line Scan and Flying Line Active IR Thermography operated with a 6-axis robot
}

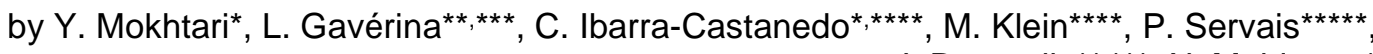 \\ J. Dumoulin ${ }^{\star \star * \star \star *}$, X. Maldague*

\begin{abstract}
* Electrical and Computer Engineering Dpt, LVSN-MIVIM, Laval University, Av. De la Médecine, Québec, Canada. ** IFSTTAR, COSYS-SII, Allée des Ponts et Chaussées, F-44344, Bouguenais, France.

*** Inria, I4S Team, Campus de Beaulieu, F-35042 Rennes, France

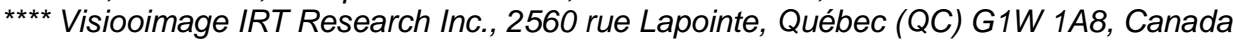

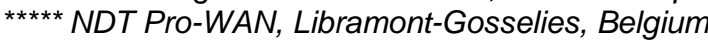

Contact authors: mohammed-yacine.mokhtari.1@ulaval.ca and ludovic.gaverina@ifsttar.fr

\begin{abstract}
In this paper, two Non Destructive Testing approaches by active infrared thermography mounted on a 6-axis robot are presented and studied. Data acquisition and thermal excitation is carried out dynamically over various CFRP specimens with increasing geometry complexity, from planar, to convex and concave shapes. An automated procedure is proposed to reconstruct thermal image sequences issued from the two scanning procedure studied: Line Scan and Flying Line procedures. Defective area detection is performed by image processing and an inverse technique based on thermal quadrupole method is used to map the depth of flaws. Results obtained are discussed and perspectives are addressed.
\end{abstract}

\section{Introduction}

The inspection of complex-shaped specimen is complicated using the conventional thermal methods, such as flash or lock-in techniques. Such investigation represents a serious challenge in particular for the aeronautic industry.

Twenty years ago, Krapez et al. [1] proposed the "flying spot technique" based on a constant displacement of the laser spot to detect cracks in steel. Later, the Pulsed Flying Spot (P.F.S) [2] was developed to obtain in-plane thermal diffusivity fields on heterogeneous and anisotropic materials. Though the laser spot is a very interesting approach due to its known analytical solution, it has a lower scanning rate than the line scan method. For this reason, the flying line method has been proposed instead to detect cracks in metals [3], [4] as well as disbonding in composite materials [5], [6].

Recently, the MIVIM laboratory at Laval University in collaboration with the company Visiooimage has developed and improved the Robotized Inspection by Thermography and Advanced processing for the inspection of aeronautical components (RITA): it is a robotic arm that allows inspection of complex-shaped specimens [8]. The goal of the present study is the thermogram reconstruction whatever the configuration of the RITA set-up, and in a second stage to calculate the depth map of defects in composite material with various shapes.

In this paper, we first introduce the test bench with the 6-Axis robot and describe the planar specimen used. Dedicated post-processing methods studied and developed to reconstruct thermal sequence acquired in line scan and flying line modes are presented and results shown. Then damage localization characterization approaches are introduced and results analysis are discussed. Finally, conclusions and perspectives are addressed.

\section{Experimental set-up and procedures}

\subsection{Active IR test bench}

The inspection of the object surface is carried out by active infrared thermography technique. The heating of specimen is performed using a linear configuration under two data acquisition modes: line scan and flying line. In line scan, both the heating source and the IR camera move synchronously over the surface of the specimen by using a robotic arm (Figure 1.a.). In flying line, only the heating source mounted on the robotic arm moves while data are acquired with the IR camera fixed on a tripod in front of the scene (Figure 2.a.). Figure 1.b and Figure 2.b show IR images of the scene at a fixed time for the two configurations.

Two IR cameras were used during experiments. A FLIR SC655 (640*480 pixels, NETD 30mK, 50 FPS, pitch 17 $\mu \mathrm{m}$, spectral band $7.5-13 \mu \mathrm{m})$ and a FLIR A65 (640*512 pixels, NETD 50mK, $30 \mathrm{FPS}$, pitch $17 \mu \mathrm{m}$, spectral band 7.5 $13 \mu \mathrm{m})$. The linear heating source is an optical one mounted horizontally on the robotic arm. The robotic arm is the RITA system developed by the MIVIM Laboratory at Laval University in collaboration with Visiooimage Inc. [8]. 
(a)

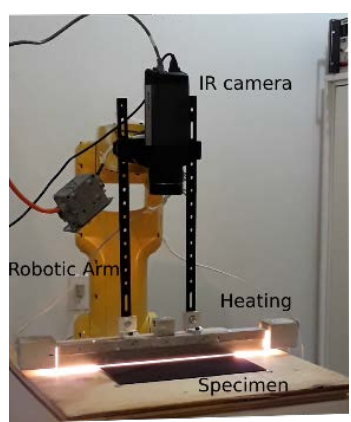

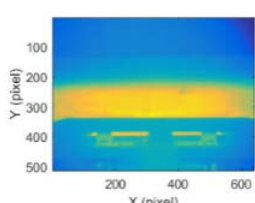
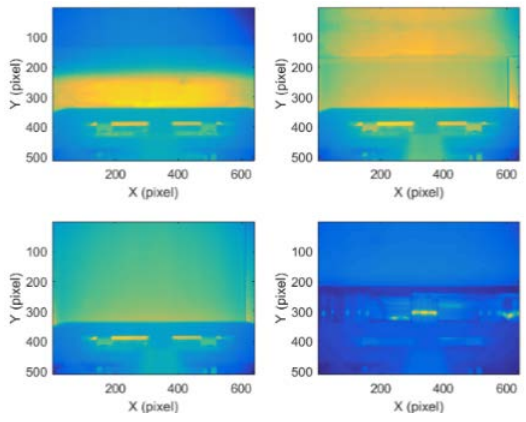

(b)

Fig. 1 Robotized line scan active thermography: (a) set-up photography; (b).IR image during the scan : $1.2 \mathrm{~s}$, $4 s, 8 s, 12 \mathrm{~s}$.

(a)

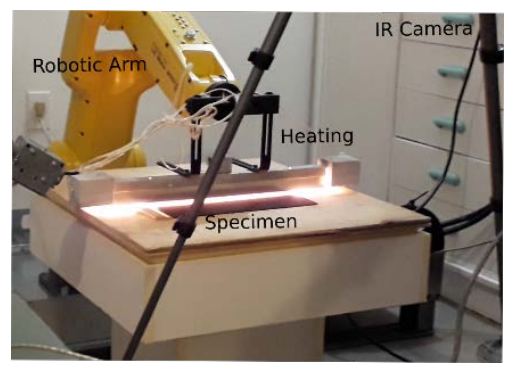

(b)
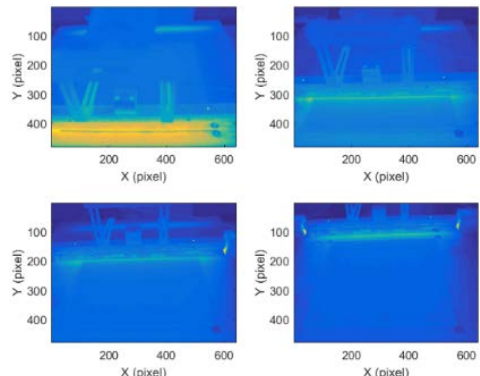

Fig. 2 Robotized flying line active thermography: (a) set-up photography; (b).IR image during the scan : $1.2 \mathrm{~s}$, $4 s, 8 s, 12 \mathrm{~s}$.

\subsection{Experimental specimen}

Experiments were carried out on academic CFRP specimens [7], which incorporate twenty-five square Teflon inserts of different sizes at different locations, as illustrated in Figure 3.

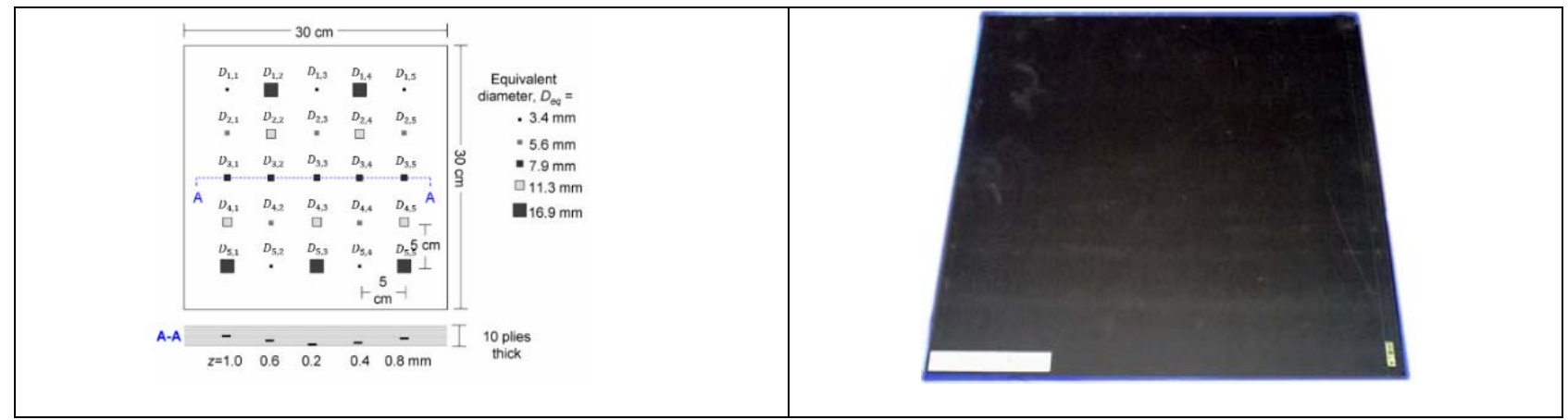

Fig. 3 Sample schematic view with defect locations (a), photography of the sample [7] (b).

The dimensions of the square CFRP sample, presented hereafter, are $300 \mathrm{~mm} * 300 * \mathrm{~mm} * 2 \mathrm{~mm}$. The thermal properties of the material are the following:

Thermal conductivity $\lambda=0.8 \mathrm{~W} \cdot \mathrm{m}^{-1} \cdot \mathrm{K}^{-1}$, volumetric heat capacity $\rho \mathrm{C}_{\mathrm{p}}=1.92^{\star} 10^{-6} \mathrm{~J} \cdot \mathrm{m}^{-3} \cdot \mathrm{K}^{-1}$ and thermal diffusivity $\alpha=4.2^{\star} 10^{-7} \mathrm{~m}^{2} \cdot \mathrm{s}^{-1}$.

\section{Reconstruction}

\subsection{Line Scan}

In the literature [8], many parameters must be known to reconstruct the thermographic matrix $I_{r}$ from line scan method such as, (i), spatiotemporal resolution (size of FPA matrix), (ii), field of view and (iii), acquisition rate. 
In our case, it is possible to reconstruct the matrix $I_{r}$ without those parameters. This algorithm is based on the tracking of each line pixel independently for each frame of the matrix $I(x, y, t)$. The pixel line $p\left(x_{n}, y_{n}, t_{n}\right)$ of the matrix $I(x, y, t)$ is shifted on the initial $p\left(x_{0}, y_{0}, t_{0}\right)$ by $y_{0}+N \cdot d_{y}$ as shown in the Figure 4.a. After determining this shift from the slope of a straight line of the maximum of each pixel line of the matrix $I(y, t)$ (Figure 5.a), each pixel line reconstructed temporally is rearranged in the new matrix $I_{r}\left(x, t, y^{\prime}\right)$ where $y^{\prime}$ is equal to $N \cdot d_{y}$ as shown in the figure 4.b.

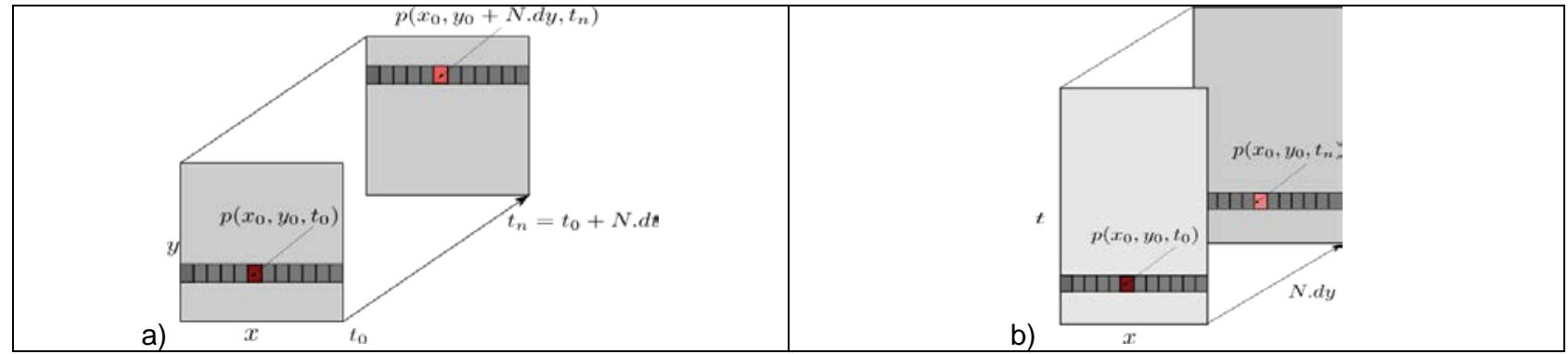

Fig. 4. Schematic reconstructed thermogram (a), raw thermogram (b). reconstructed thermogram

An example of thermographic image reconstructed is shown in figure 5.b from the dynamic matrix.

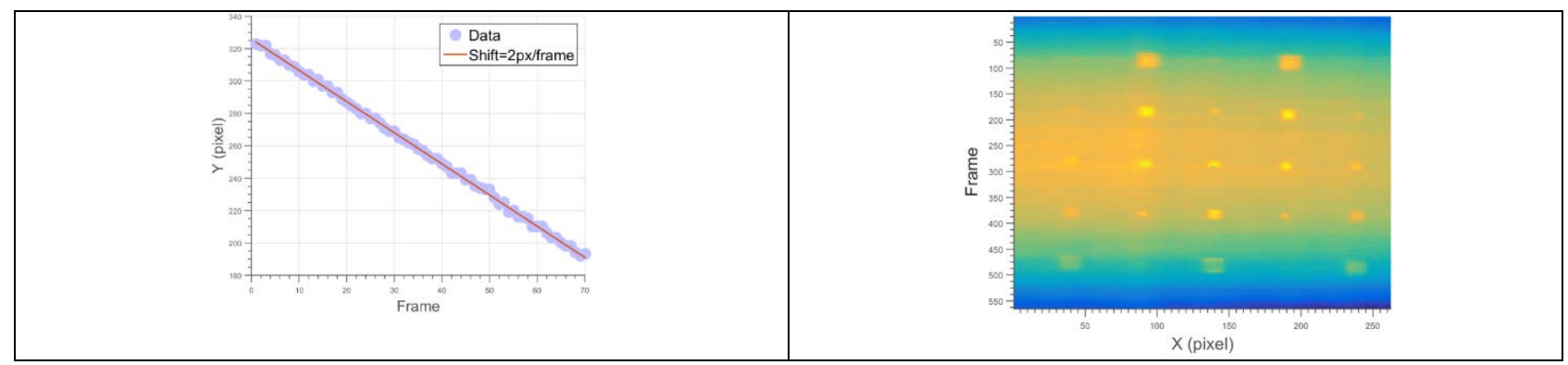

Fig. 5. (a), Estimation of the shift from the slope of the curve of the maximum of each pixel line of the matrix $I(y, t),(b)$. reconstructed thermogram.

The presence of the defects is clearly seen, thus algorithm can be used to reconstruct the raw thermogram. It can also be observed the non-uniform heating in the background, which reduces defective area thermal contrast.

\subsection{Flying line}

In the flying line configuration, the algorithm proposed to reconstruct the matrix $I_{r}$ is faster and simpler than the previous method. In this case, the camera frame rate is not synchronized with the displacement of the robotic arm. As the previous algorithm each line pixel is heating independently for each frame of the matrix $I(x, y, t)$. However, the pixel line corresponding to the $p\left(x_{0}, y_{0}, t_{0}\right)$ is the pixel line $p\left(x_{0}, y_{0}, t_{n}\right)$ as show in Figure $6 . \mathrm{a}$ and $\mathrm{b}$. Therefore, the method to reconstruct the new infrared sequence $I_{r}(x, y, t)$ is based on the tracking of the gradient for each frame. In this new infrared sequence $I_{r}(x, y, t)$, there is a time-offset between each pixel line from each frame as show in Figure 6.c. This time-offset is estimated for each pixel line by detecting the barycentre at each frame as it is shown in the Figure 6.d. The new thermal sequence obtained is similar to the ones typically observed in a static flash (i.e. short pulse) method [9]. 


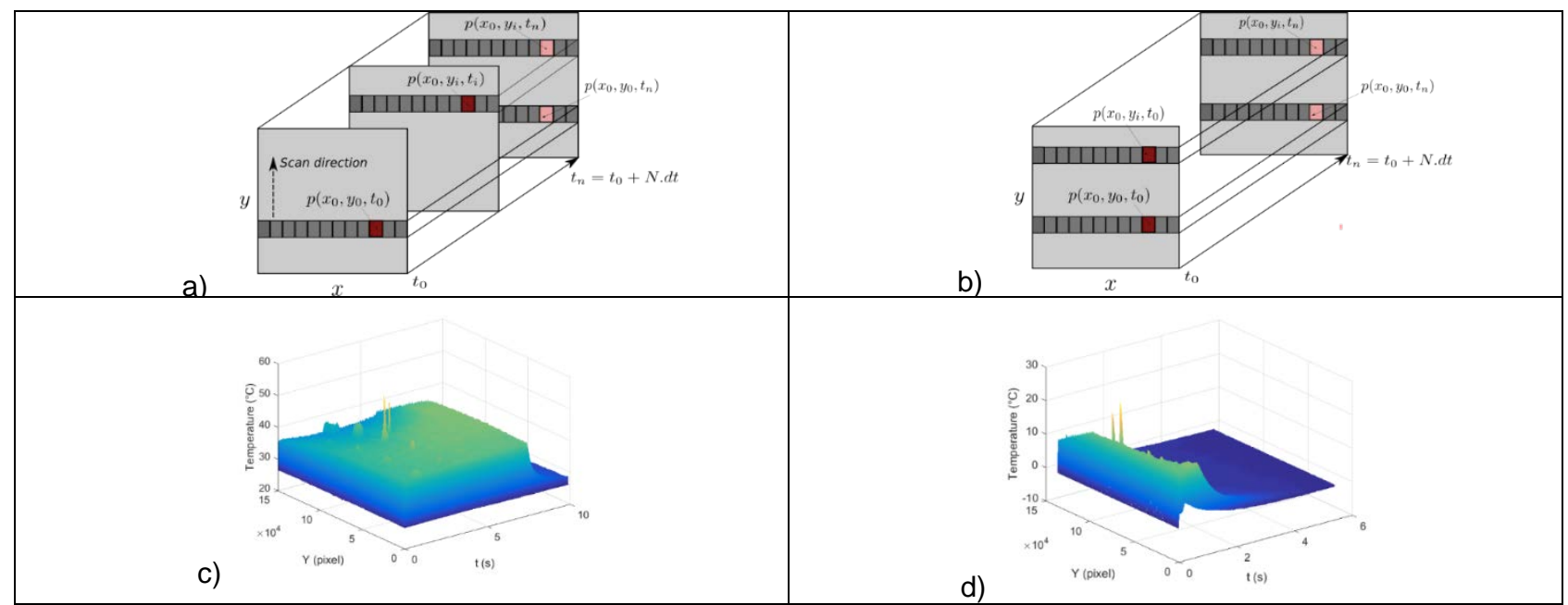

Fig. 6. Schematic reconstructed thermogram (a), raw thermogram (b), reconstructed thermogram, $I_{r}(y, x \cdot t)$ represents themal profile: (c), shifted between each frame, (d); not shifted between each frame.

An example of a thermographic image reconstructed from the dynamic matrix $I(x, y, t)$ is shown in figure 7 .

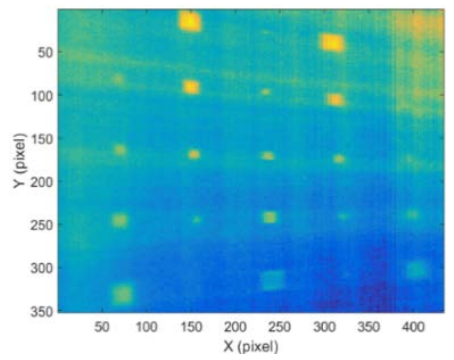

Fig. 7. Averaged thermogram over several hundreds of reconstructed thermograms corresponding to flying line scan active thermography

The presence of the defects is clearly seen, thus algorithm can be used to reconstruct the raw thermogram. One can also observed a deformation in the reconstructed thermal image due to the non-regular initial field of view of the camera. This can be a problem for the future estimation due to the non-constant spatial sampling. In any case, several methods may be used to attenuate effects of such problem [10].

\section{Defect localization and characterization}

\subsection{Thermal signal filtering}

For line scan mode, Figure 8.b shows the thermal profiles for defective areas at the same depth $\left(D_{1,2}, D_{1,4}\right)$ and sound area $\left(S_{1,2}, S_{1,4}\right)$ obtained from line scan thermograms. It can be observed for these profiles a similar thermal contrast between defect and sound areas, and the decay of these curves is similar to a static pulsed thermography [9].

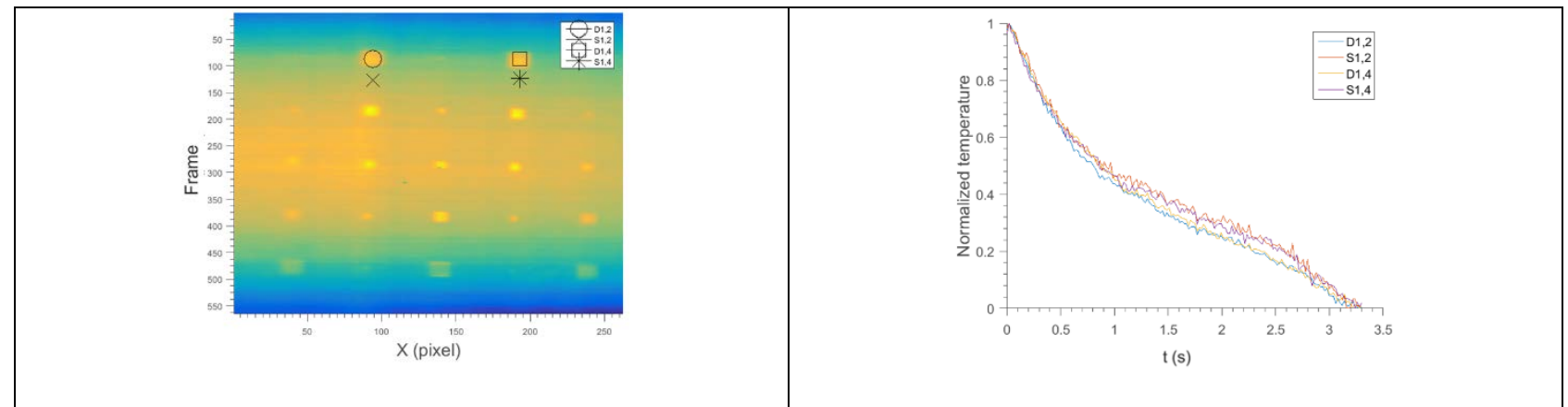

Fig. 8. Averaged thermogram over several hundreds of reconstructed thermograms corresponding line scan active thermography: (a) raw reconstructed thermography; (b) thermal profiles. 
In flying line mode, Figure 9.a shows the thermal profiles for defect areas at the same depth $\left(D_{1,4}, D_{2,4}, D_{3,4}, D_{4,4}\right)$ and Figure 9.b shows thermal profiles for different depth $\left(D_{3,1}, D_{3,2}, D_{3,3}, D_{3,4}\right)$. As can be seen, these profiles produce $a$ positive thermal contrast with respect to the sound area and the decay depends on the depth. These last thermal profiles show that flying line experiment after reconstruction is similar to thermal profile from static flash method [9].

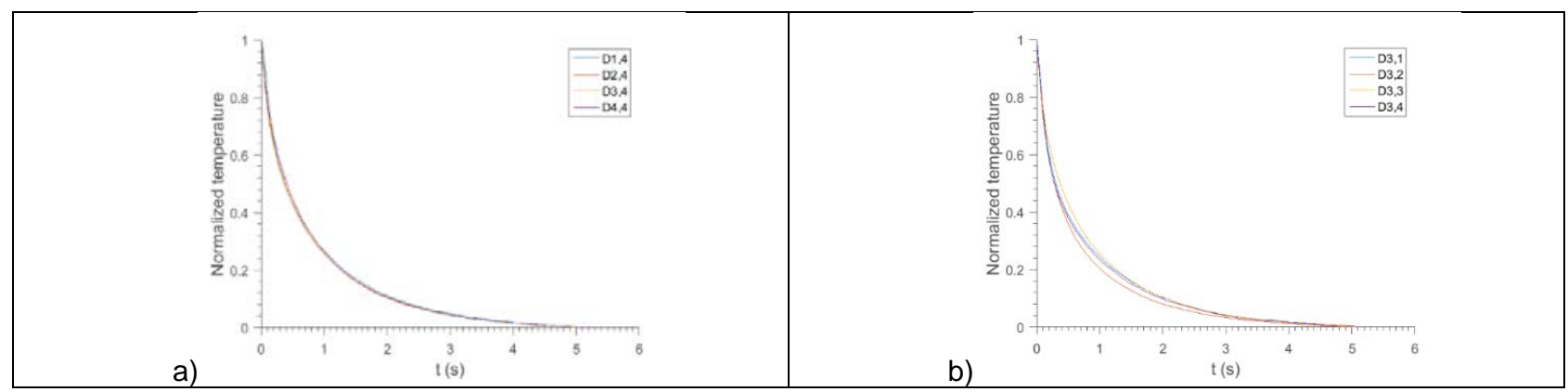

Fig. 9. Flying line scan active thermography: (a) thermal profiles: defects at the same depth and (b) defects not the same depth.

Some additional observations can be made from these results. The raw reconstructed thermograms in Figures 10.a and c present some signs of non-uniform heating, which reduce the defect contrast. A correction stage may be added to the raw reconstructed thermogram to increase the defect contrast. For instance, here we use polynomial fitting to approximate thermal background and correct thermograms. However, some artefacts are still present in the raw reconstructed thermogram due to some discrete inhomogeneities inherent to the heating source, as it is shown in Figures $10 . b$ and $10 \mathrm{~d}$.



Fig. 10. Flying line active thermography: (a), non-uniform heating, (b), background corrected. Line scan active thermography: (c), non-uniform heating, (d), background corrected.

\subsection{Defect localization by Singular Value Decomposition (S.V.D)}

Since several years, Singular Value Decomposition (S.V.D) is applied in image processing and signal processing problems for data compression and noise reduction. In Non Destructive Testing and thermal analysis, SVD-based methods have been employed to defect detection [11]. In this part, the Singular Value Decomposition (S.V.D) is applied to the previous results from line scan and flying line. follows:

For that, the thermogram matrix (3D) representing space and time has to be reorganized as a $2 \mathrm{D}$ matrix as 


$$
T(X, t)=T(x, y, t)
$$

Applying S.V.D on a thermal field of 2D thermogram matrix is reported in equation 2:

$$
T(X, t)=\sum_{k=1}^{\min \left(N_{x}, N_{y}, N_{t}\right)} \gamma_{k} U_{k}(X) V_{k}(t)^{t}
$$

Where

$U_{k}(X)$ and $V_{k}(t)$ are orthogonal functions and $\gamma_{k}$ are the singulars values in decreasing mode.

The columns of the matrix $U$ represent the empirical orthogonal functions $(E O F)$ that describe the spatial variations of data [12]. The first columns of the matrix $U$ represent the most characteristic variability of the data. The figure 11 which represent the logarithm of singulars values from S.V.D apply to the matrix $T(X, t)$ as a function of singular values index show the first three singulars values is much greater than the others.

These columns of the matrix $U$ has to be reorganized as a 2D matrix as follows:

$$
\operatorname{EOF}_{k}(x, y)=\sum_{k=1}^{k=3} U_{k}(X)
$$

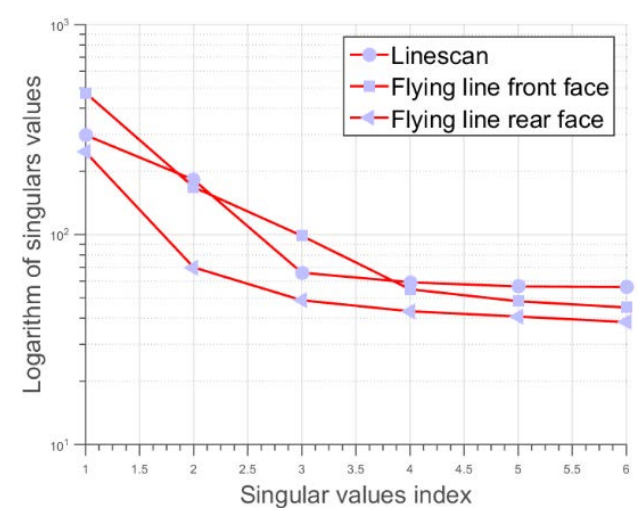

Fig. 11. Diagonal of singular values related to the expression

So the original data can be represented only with three $E O F$.

In figure 12, sound and faulty areas were localized by using EOF maps for line scan, flying line for front and rear face scan. This method can be used to localize the spatial position of defect.
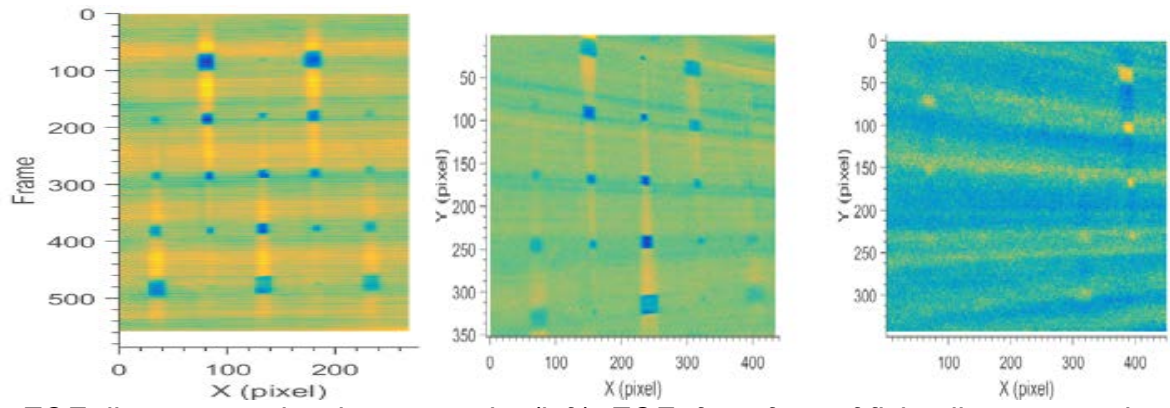

Fig. 12. PCT results: $E O F_{2}$ line scan active thermography (left), EOF 1 front face of flying line scan active thermography (middle), and EOF 1 front rear of flying line scan active thermography (right)

\subsection{Thermal contrast}

The thermal contrast is a processing technique to enhance subsurface defect visibility and also a quantitative method to estimate defect depth and size [13-14]. This method requires a priori information, such as, sound area and defective area localization. However, these information is not always available but it is possible to apply the previous method (see section 3.1) to identify them. In this case, the sound area is not uniform all over the specimen.

The absolute thermal contrast definition is reported below:

$$
C_{T}(i, j, t)=T_{d}(i, j, t)-T_{S}(i, j, t)
$$


where $T_{d}(i, j, t)$ defect area and $T_{s}(i, j, t)$ sound area at location $i, j$.

Results obtained by calculating absolute thermal contrast for both experiments (line scan and flying line) are presented in Figures 13a and 14a. Amplitude differences in thermal contrast maps (i.e. profiles) are observed. Thermal contrast from line scan is more significant than the flying line because there is no initial field of view induced effect on reconstructed thermal images.

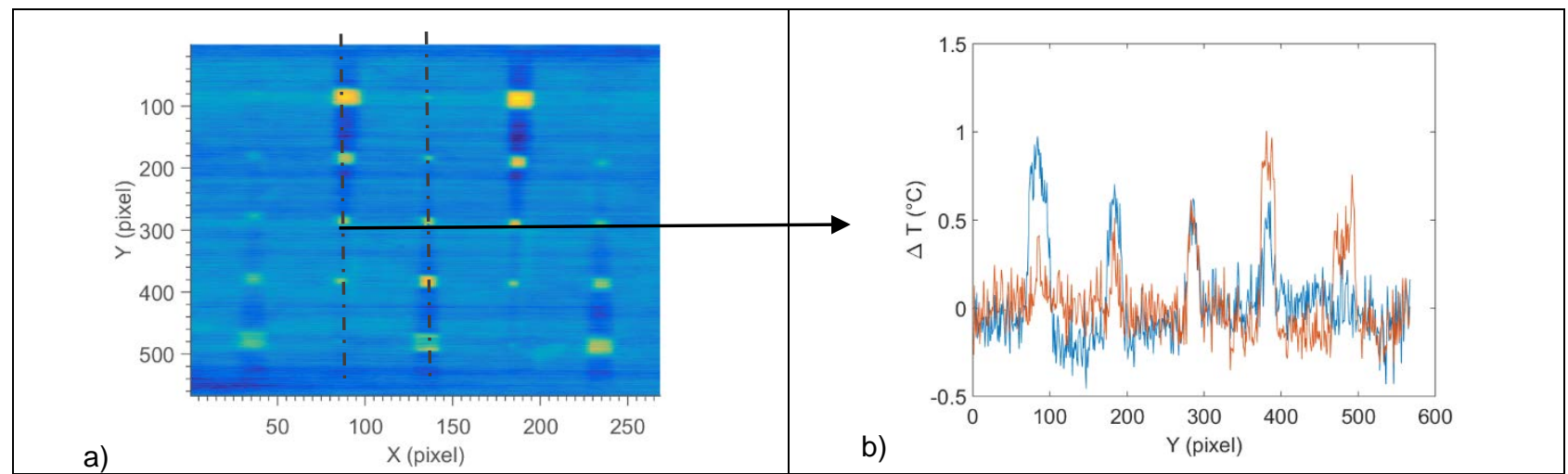

Fig. 13. Line scan active thermography: (a), thermal contrast map at a fixed time, (b), thermal profiles.

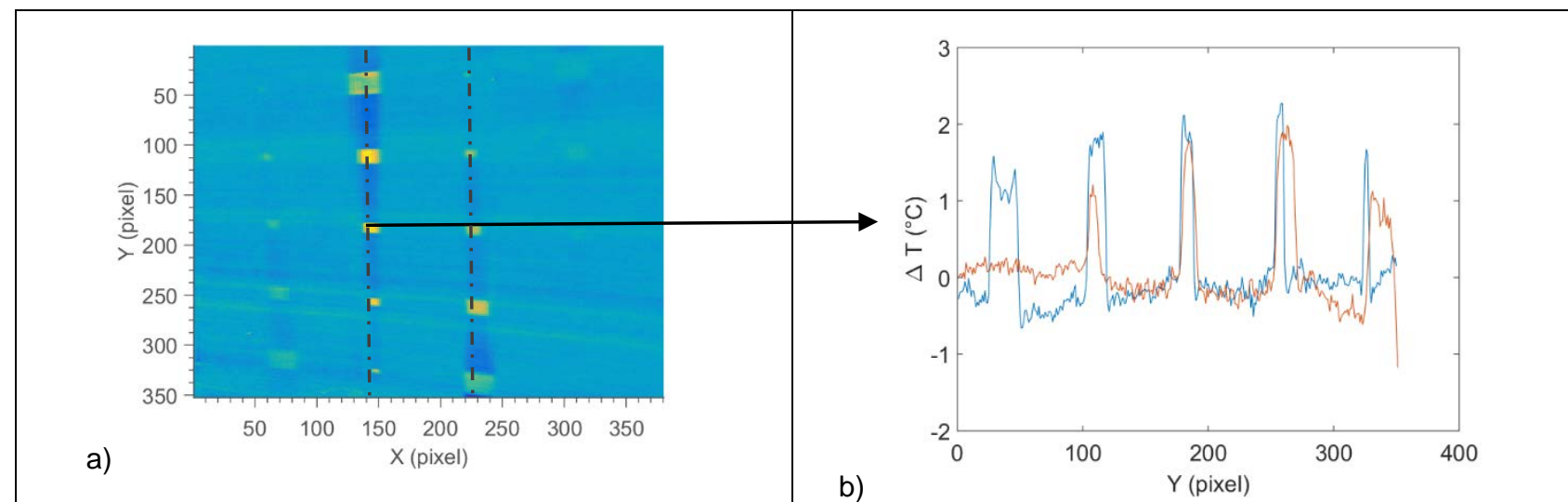

Fig. 14. Flying line active thermography: (a), thermal contrast map at a fixed time, (b) thermal profiles.

Figure 15 shows thermal signal as a function of time obtained from both scans: line scan (Figure 15 a) and flying line (Figure $15 \mathrm{~b}$ ). These figures illustrate thermal contrast decay with defect depth. Furthermore, for the chosen scan speed maximum contrats may appear or not appear in the time interval of observation, depending also on defect depth.

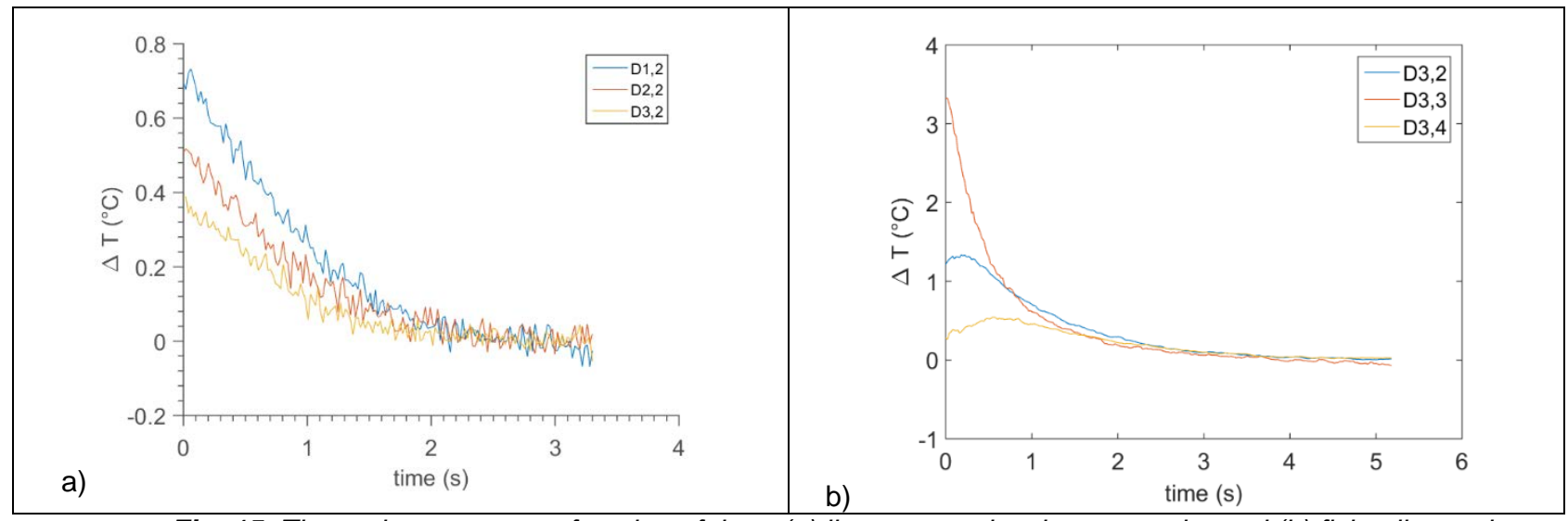

Fig. 15. Thermal contrast as a function of time: (a) line scan active thermography and (b) flying line active thermography 
In such context using time of the appearance of the maximum thermal contrast for defect depth retrieval will require to act on the robot scan speed. A possible alternative is to use thermal inverse method to retrieve such information.

\subsection{Thermal Inverse approach}

\subsubsection{Direct thermal model}

In this section, we first apply the quadrupole method to calculate the surface temperature of multi-layered CFRP, supposed to have homogeneous thermal properties, that is illuminated by uniform radiative source. Accordingly, in this simple configuration the one-dimensional approach can be used. The quadrupole formalism expresses, for each layer $i$, the upstream temperature flux vector in terms of downstream temperature flux vector in Laplace space.

Considering a parallelepiped specimen with a thickness $e(2 \mathrm{~mm})$, a thermal conductivity $k(0.8 \mathrm{~W} / \mathrm{mK})$, a volumetric mass density $\rho\left(1600 \mathrm{~kg} / \mathrm{m}^{3}\right)$, a heat capacity $c_{p}(1200 \mathrm{~J} / \mathrm{Kg} / \mathrm{K})$ and a thermal diffusivty $\left(4.2^{\star} 10^{-7} \mathrm{~m} / \mathrm{s}\right)$. The specimen is submitted to heat exchange both on front $h_{1}$ and rear face $h_{2}$.

The specimen is not homogeneous due to delamination (defective areas), so we have considered two models such as: (i), sound area with one layer and (ii), defect area with two layers separated by the defect whose is modelled as follows:

(i) Quadrupole formalism for sound area

$$
\left[\begin{array}{l}
\theta_{1} \\
\varphi_{1}
\end{array}\right]=\left[\begin{array}{ll}
1 & 0 \\
h_{1} & 1
\end{array}\right]\left[\begin{array}{ll}
A_{s} & B_{S} \\
C_{s} & D_{s}
\end{array}\right]\left[\begin{array}{cc}
1 & 0 \\
h_{2} & 1
\end{array}\right]\left[\begin{array}{l}
\theta_{2} \\
\varphi_{2}
\end{array}\right]
$$

After algebraic manipulation, the Laplace temperature over front face for a sound area can be written as:

$$
\theta_{2}=\frac{\varphi_{1}\left(A+B h_{2}\right)+\varphi_{2}(D A-B C)}{C+D h_{2}+A h_{1}+B h_{1} h_{2}}
$$

Quadrupole formalism for defective area

$$
\left[\begin{array}{l}
\theta_{1} \\
\varphi_{1}
\end{array}\right]=\left[\begin{array}{cc}
1 & 0 \\
h_{1} & 1
\end{array}\right]\left[\begin{array}{ll}
A_{1} & B_{1} \\
C_{1} & D_{1}
\end{array}\right]\left[\begin{array}{cc}
1 & R \\
0 & 1
\end{array}\right]\left[\begin{array}{ll}
A_{2} & B_{2} \\
C_{2} & D_{2}
\end{array}\right]\left[\begin{array}{cc}
1 & 0 \\
h_{2} & 1
\end{array}\right]\left[\begin{array}{l}
\theta_{2} \\
\varphi_{2}
\end{array}\right]
$$

with:

$$
A_{i}=D_{i}=\cosh \left(\sqrt{\frac{p}{a_{i}}} e_{i}\right) ; B_{i}=\frac{\sinh \left(\sqrt{\frac{p}{a_{i}}} e_{i}\right)}{\lambda_{i} \sqrt{\frac{p}{a_{i}}}} ; C_{i}=\lambda_{i} \sqrt{\frac{p}{a_{i}}} \sinh \left(\sqrt{\frac{p}{a_{i}}} e_{i}\right)
$$

Where $p$ is the Laplace variable and $R=\frac{e}{\lambda}$ the thermal resistance considered between two layers.

The main purpose is to be able to characterize the depth and the thermal resistance of defects. Based on the one-dimensional thermal model (Eq. (6)), an inverse problem is solved using the Levenberg-Marquardt algorithm, that is applied independently to each pixel of the 3D thermogram matrix.

As the first and second layer are constituted of an orthotropic material and as there is a priori knowledge on the thermophysical and thickness properties of the CFRP, the number of unknown parameter is reduced in the inverse model. The parameters estimated are then the thermal resistance $R$ and the depth of defect, which is obtained from the thickness of two layers $e_{1}$ and $e_{2}$.

Furthermore, the energy deposed on the surface is estimated from the first model (Eq. (4)) using pixels without defect and considering a square wave form excitation as described in Eq. (8). The heat duration $\tau$ is related to the displacement velocity of the heating line during the experiments (Figure 16. a). After having reconstructed the thermogram from the flying line scan, it is still not to possible to have the shape evolution related to the heat duration (Figure 16. b). Hence, in that case, we have to consider $\tau$ that is equal to the displacement duration of the heating (3s in the shown results).

The Laplace expression of the heating source is:

$$
\varphi_{1}=\frac{Q}{p}\left(1-e^{-\tau p}\right)
$$




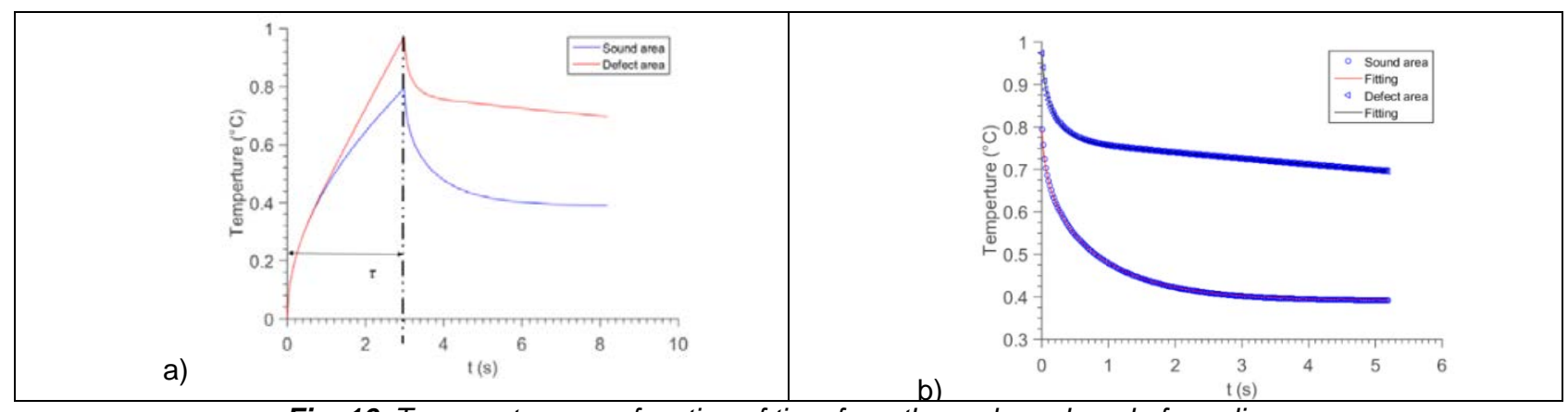

Fig. 16. Temperature as a function of time from thermal quadrupole formalism.

\subsubsection{Depth estimation}

In this part, the estimated parameter associated to front surface observation of the CFRP plate are presented for flying line mode. Figure 17 shows that the estimation procedure applied to the reconstructed thermal infrared image sequence is able to characterize defect depth in CFRP material. However, the spatial distortion due to the field of view of the IR camera fixed on a tripod in front of the scene affect the depth estimation. In order to correct this problem, it could be possible to apply an algorithm to recover a non-projective view [10].

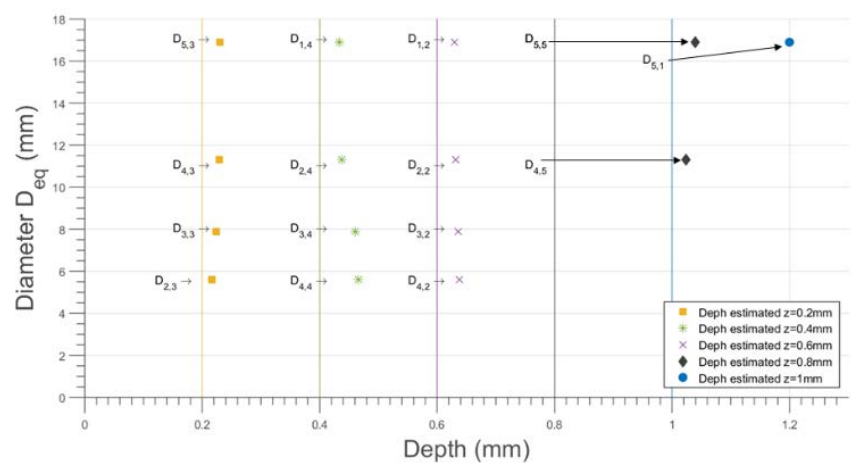

Fig. 17. Diameter as function of the depth estimation (symbol) for each defect of CFRP specimen. The real depth is representing by a coloured straight line.

Moreover, it can be observed that for a given scan speed, depth estimation depends on the real depth and size of defect, but also on the validity of the 1D inverse model as suggested in [15] in their sensitivity analysis.

\section{Conclusion and perspectives}

In this paper, two kind of active infrared non destructive testing processes, mounted on a 6-axis robot, have been studied: line scan and flying line. Thermographic matrix have been reconstructed from raw data using dedicated processing approaches. SVD processing have been implemented for defect localization and combined with an inverse method to characterize defective areas.

It has been proven that the profile follow a temperature decay similar to the ones observed in a pulsed thermography. Therefore, a one dimensional thermal quadrupole model with an estimation procedure has been implemented to estimate the depth of the defect. This model represents rough simplification of the actual physical problem, such as lateral diffusion. The Levenberg-Marquadt algorithm has been used for the minimization procedure for each pixel. This minimization procedure apply to infrared image reconstructed (434*352 pixels) may take more $90 \mathrm{~h}$ on a standard Labtop computer to obtain a Thickness Map. However, it should be possible to improve drastically the computation time (< some hours) by an efficient GPU (Graphical Processing Unit) during the implementation of parameter estimation. The estimation procedure for each pixel of infrared image reconstructed could be assigned to one thread block in the GPU.

Future work will address a series of complete scans of different academic shape panels using also transmission scanning and associated signal processing. Improvement in inverse model will be also studied to address three dimensional thermal phenomenon observed during some experiments.

\section{Acknowledgment}

Authors wish to thanks the Collaborative Research and Training Experience (CREATE) of the National Sciences and Engineering Research Council of Canada (NSERC) for supporting this work carried out in the framework of oN DuTy! Program. NSERC Discovery and Canada research chair programs are also acknowledged. 


\section{REFERENCES}

[1] J.-C. Krapez, “Résolution spatiale de la caméra thermique à source volante,” Int. J. Therm. Sci., vol. 38, no. 9, pp. 769-779, 1999.

[2] L. Gaverina, J. C. Batsale, A. Sommier, and C. Pradere, "Pulsed flying spot with the logarithmic parabolas method for the estimation of in-plane thermal diffusivity fields on heterogeneous and anisotropic materials,” J. Appl. Phys., vol. 121, no. 11, p. 115105, 2017.

[3] T. Li, D. P. Almond, and D. A. S. Rees, "Crack imaging by scanning laser-line thermography and laser-spot thermography,” Meas. Sci. Technol., vol. 22, no. 3, p. 035701, 2011.

[4] N. Rajic, "Modelling of thermal line scanning for the inspection of delamination in composites and cracking in metals," DEFENCE SCIENCE AND TECHNOLOGY ORGANISATION VICTORIA (AUSTRALIA) PLATFORM SCIENCES LAB, 2004.

[5] L.-D. Théroux, J. Dumoulin, and J.-L. Manceau, "Dynamic heating control by infrared thermography of prepreg thermoplastic CFRP designed for reinforced concrete strengthening," in 12th International Conference on Quantitative InfraRed Thermography, 2014.

[6] L.-D. Théroux, J. Dumoulin, and E. Merliot, "Automatic installation of thermoplastic CFRP monitored by infrared thermography for pipelines,” Adv. Infrared Technol. Appl., p. 24, 2015.

[7] C. Ibarra-Castanedo and X. P. Maldague, "Pulsed phase thermography inversion procedure using normalized parameters to account for defect size variations,” in Proc. SPIE, 2005, vol. 5782, pp. 334-341.

[8] C. Ibarra-Castanedo, P. Servais, A. Ziadi, M. Klein, and X. Maldague, "RITA-Robotized Inspection by Thermography and Advanced processing for the inspection of aeronautical components," in 12th International Conference on Quantitative InfraRed Thermography, 2014.

[9] W. J. Parker, R. J. Jenkins, C. P. Butler, and G. L. Abbott, "Flash method of determining thermal diffusivity, heat capacity, and thermal conductivity,” J. Appl. Phys., vol. 32, no. 9, pp. 1679$1684,1961$.

[10] N. Le Touz, T. Toullier, and J. Dumoulin, "Infrared thermography applied to the study of heated and solar pavement: from numerical modeling to small scale laboratory experiments," in Thermosense: Thermal Infrared Applications XXXIX, 2017, vol. 10214, p. 1021413.

[11] N. Rajic, "Principal component thermography," DEFENCE SCIENCE AND TECHNOLOGY ORGANISATION VICTORIA (AUSTRALIA) AERONAUTICAL AND MARITIME RESEARCH LAB, 2002.

[12] C. Ibarra-Castanedo, A. Bendada, and X. Maldague, “Thermographic image processing for NDT,” in IV Conferencia Panamericana de END, 2007, vol. 79.

[13] J. C. Krapez, F. Lepoutre, and D. Balageas, "Early detection of thermal contrast in pulsed stimulated thermography,” NDT E Int., vol. 6, no. 29, p. 393, 1996.

[14] X. Maldague, "Theory and practice of infrared technology for non-destructive testing", John Wiley \& sons Inc., 2001.vier

[15] A. Crinière, J. Dumoulin, C. Ibarra-Castanedo, X. Maldague, "Inverse model for defect characterization of externally glued CFRP on reinforced concrete structures: Comparative study of square pulsed and pulsed thermography", Quantitative InfraRed Thermography Journal, Taylor \& Francis Editor, vol 11, pp 84-114, 2014. 